C2016 IEEE. Personal use of this material is permitted. Permission from IEEE must be obtained for all other uses, in any current or future media, including reprinting/republishing this material for advertising or promotional purposes, creating new collective works, for resale or redistribution to servers or lists, or reuse of any copyrighted component of this work in other works. This is the author's version of an article that has been published in the conference proceedings. The final version of record is available at https://doi.org/10.1109/PLANS.2016.7479725 


\title{
Multipath Assisted Positioning for Pedestrians using LTE Signals
}

\author{
Markus Ulmschneider and Christian Gentner \\ German Aerospace Center (DLR) \\ Institute of Communications and Navigation \\ Oberpfaffenhofen, Münchner Strasse 20, 82234 Wessling, Germany \\ \{markus.ulmschneider, christian.gentner\}edlr.de
}

\begin{abstract}
The rapid growth of available services depending on location awareness has led to a more and more increasing demand for positioning in challenging environments. Global navigation satellite system (GNSS) based positioning methods may fail or show weak performance in indoor and urban scenarios due to blocking of the signals and multipath propagation. In contrast, cellular radio signals provide better reception in these scenarios due to a much higher transmit power. Also, they offer high coverage in most urban areas. However, they also undergo multipath propagation, which deteriorates the positioning performance. In addition, there are often only one or two base stations within communication range of the user. Both of these problems can be solved by means of a multipath-assisted positioning approach. The idea is to exploit multipath components (MPCs) arriving at the receiver via multiple paths due to scattering or reflections. Such approaches highly depend on the ability to resolve the MPCs at the receiver. This is why multipath-assisted positioning schemes typically assume ultra-wideband systems. Today's cellular radio systems work with much smaller bandwidths, though. The 3rd Generation Partnership Project (3GPP) Long Term Evolution (LTE) standard uses bandwidths up to $20 \mathrm{MHz}$. The aim of this paper is to show by means of measurements that multipathassisted positioning is possible using 3GPP-LTE signals with only two base stations. We apply an advanced signal processing algorithm to track MPCs arriving at the mobile terminal, and to estimate the position of the mobile terminal. Since each of the MPCs can be regarded as being sent from some physical or virtual transmitter, we estimate the positions of transmitters in addition. Assuming only the starting position and direction of the mobile terminal to be known, the results show that the root mean square positioning error of the mobile terminal is always below 1.8 meters. In $90 \%$ of the cases, it is below 1.25 meters.
\end{abstract}

\section{INTRODUCTION}

Positioning approaches using global navigation satellite systems (GNSSs) show weak performance in situations with bad view-to-sky conditions, such as indoors or in urban areas. However, the amount of services based on location awareness has drastically increased over the past years, particularly in such scenarios. Examples for these services range from classical navigation over geotagging to automated emergency calls. This has led to more and more research efforts using signals of opportunity (SoO) for self-localization. Multipath propagation has been considered a problem for position estimation with standard algorithms such as the delay locked loop, as it biases range estimates. Most of the standard positioning approaches estimate the channel impulse response (CIR) and try to remove

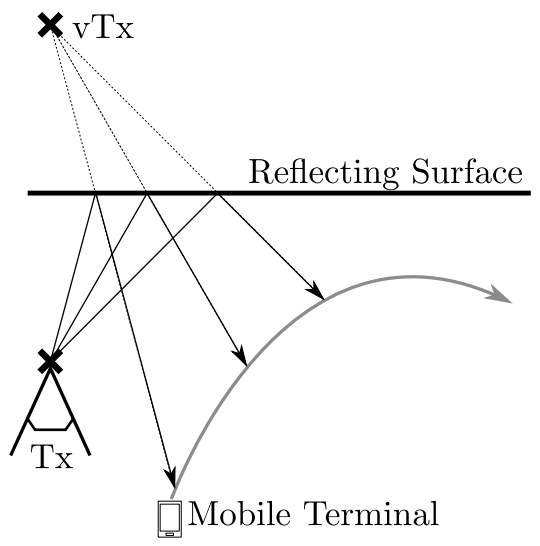

Fig. 1. Signals from the physical transmitter Tx are reflected at the straight surface and can be interpreted as originating from a virtual transmitter vTx, which is static during the receiver motion. The position of vTx is the position of the physical transmitter Tx mirrored at the surface.

the influence of multipath components (MPCs) on the line-ofsight (LoS) path.

The idea of multipath-assisted positioning is contrary. Assuming one transmitter, multiple MPCs arise due to reflections and scattering of the electromagnetic signal. The fundamental principle is to treat each obtained MPC at the receiver as a SoO being broadcast from some physical or virtual transmitter. This idea is illustrated in Fig. 1 the signals broadcast by the physical transmitter Tx are reflected at the surface. For the mobile terminal, these reflected signals seem to be emerging from the virtual transmitter vTx. The position of the virtual transmitter is the same for all shown positions of the mobile terminal, if the physical transmitter and the reflecting surface are static.

The virtual transmitters can act as additional base stations and be used by a receiver for localization if their positions are known. Hence, locating the receiver might be possible if only one physical transmitter is present, and this physical transmitter does not have to be in LoS to the receiver. The location of these transmitters might be known if a-priori information is available. This could be the position of a physical transmitter together with a floor plan in the indoor case, or the location of surrounding structures in the outdoor 
case, such as buildings or environmental landmarks. Then, the location of the virtual transmitters can be obtained by means of geometrical considerations.

In [1], the authors propose a multipath assisted indoor tracking scheme, where a floor plan is known. A similar principle is applied in [2], where the structure of surrounding walls is known for tracking a target for a radar application. In [3], a simple urban multipath scenario is emulated using LTE signals, and a mobile terminal is tracked through the scenario. The positions of the physical and virtual transmitters is assumed to be known. The authors of [4] mainly focus on the problem of association between known physical scattering objects and impinging MPCs.

In a more general setting however, the position of the transmitters is unknown. Therefore, the authors of [5] and [6] developed an algorithm which estimates not only the position of a receiver, but also the positions of physical and virtual transmitters. The algorithm was named ChannelSLAM, since it is a simultaneous localization and mapping (SLAM) approach based on the CIRs estimated at the receiver. The location of the receiver is tracked over time, and a map in terms of the positions of the transmitters is estimated.

The Channel-SLAM algorithm does not differentiate between physical and virtual transmitters: each incoming MPC at the receiver is regarded as a SoO emerging from some transmitter in LoS, which can be physical or virtual. All transmitters are assumed to be static, which is true if the physical transmitters and the structures that reflect or scatter the signal are static. The receiver is assumed to be dynamic and to move through the scenario. Transmitters, i.e., MPCs at the receiver, might arise and vanish as the receiver moves depending on the scenario, and only transmitters that are visible for a sufficiently long time span can contribute well to the scheme.

The ability to resolve the single MPCs at the receiver is of crucial importance in multipath-assisted positioning. If two MPCs arrive at the receiver with a very small relative delay, it is difficult to resolve them with standard and even with superresolution methods. This problem becomes more drastic the smaller the bandwidth of the used signaling system is. This is why most research papers in multipath-assisted positioning assume signaling systems of high bandwidths of at least 100 $\mathrm{MHz}$ up to several $\mathrm{GHz}$.

Though, such systems have to be installed on top of existing systems, and the admissible power is limited by rules of national or international regulation authorities. Within this paper, we aim to use an existing and widely available infrastructure, namely a 3rd Generation Partnership Project (3GPP) Long Term Evolution (LTE) signaling system. A big advantage of 3GPP-LTE signals over GNSSs is their high signal power leading to a very good coverage in urban and most indoor situations. The 3GPP-LTE standard [7] defines optional signals, so called positioning reference signals (PRSs), for positioning within a 3GPP-LTE network, making 3GPP-LTE signals very interesting for positioning.

3GPP-LTE introduces various positioning techniques, of which observed time difference of arrival (oTDoA) tends to be the most accurate in bad view-to-sky conditions. It is based on relative delays of downlink signals at a mobile terminal, and the position estimation itself is performed by the 3GPP-LTE network. Thus, for positioning with oTDoA in two dimensions, there are at least four LTE base stations within communication range of the user necessary, which is unrealistic in many scenarios. In indoor scenarios and urban canyons, multipath propagation will bias the position estimate. In addition, no external measurement data, such as from an inertial measurement unit (IMU) in the mobile phone, is incorporated.

The Channel-SLAM algorithm can be used to overcome these problems at the expense of the relatively low bandwidths of at most $20 \mathrm{MHz}$ specified by LTE. Therefore, we developed a signal processing algorithm for resolving MPCs at the receiver. In outdoor measurements we apply our algorithm to track a receiver in a simple outdoor scenario, and simultaneously locate the physical and virtual 3GPP-LTE transmitters. We make use of the PRSs of the 3GPP-LTE system, which use the maximum 3GPP-LTE bandwidth of $20 \mathrm{MHz}$. In our scenario, we have only two physical transmitters. As in a real 3GPP-LTE system, we assume no synchronization of the user to the base stations. Though, we do incorporate heading information from an IMU carried by the mobile terminal to improve the tracking performance.

The main contribution of this paper is to show that even under the constraint of a bandwidth as low as $20 \mathrm{MHz}$ in a 3GPP-LTE signaling system, the resolution of the single MPCs at the receiver is still possible, and hence simultaneous tracking of a mobile terminal and mapping of transmitters can be achieved.

This paper is organized as follows: the system model as well as the used methods and algorithms are introduced in Section [II Section III explains the measurement setup and presents the measurement results. Finally, Section IV concludes the paper.

\section{System Model}

We use a two-step approach for tracking the location of the mobile terminal. In a first step, the parameters of the MPCs impinging at the mobile terminal are estimated. These estimates are used in a second step, where the positions of the mobile terminal and the transmitters are estimated by means of the Channel-SLAM algorithm. In addition, in the second step, heading information from an IMU carried by the mobile terminal is exploited. Fig. 2 gives an overview of the estimation procedure. In both steps, the estimators are based on recursive Bayesian estimation.

\section{A. Virtual Transmitters}

Fig. 1 shows a scenario where a mobile terminal receives signals from a physical transmitter that are reflected at a straight reflecting surface. As the receiver moves on its trajectory, these signals always seem to be emitted from the virtual transmitter vTx. Simple geometric considerations show 


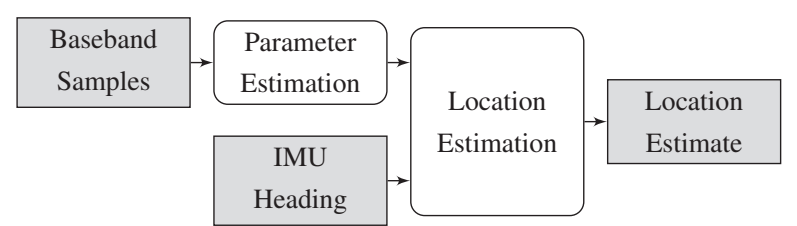

Fig. 2. Based on the recorded baseband samples, the parameters of the MPCs are estimated in the first step. In the second step, the estimates are fused with heading information from an IMU, and the positions of the mobile terminal and the virtual transmitters are estimated.

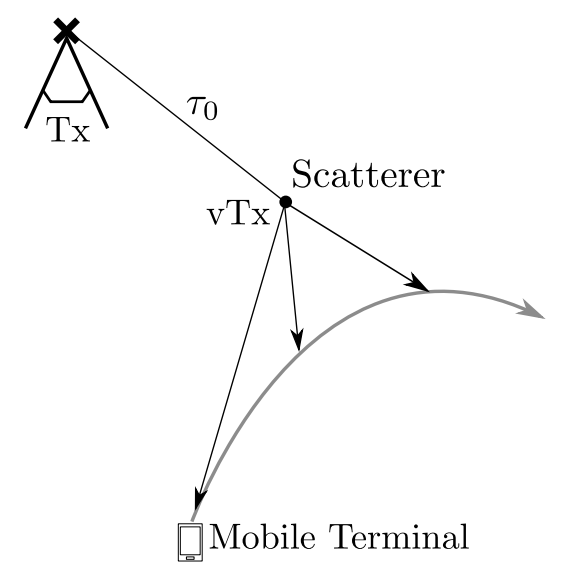

Fig. 3. Signals from the physical transmitter Tx are scattered at the punctual scatterer and can be interpreted as originating from a virtual transmitter vTx, which is static during the receiver motion. The virtual transmitter has a clock offset of $\tau_{0}$ compared to the physical transmitter Tx.

that the position of the virtual transmitter is the position of the physical transmitter mirrored at the reflecting surface [5]. Hence, if the surface and the physical transmitter are static, the virtual transmitter is as well. In addition, the physical and the virtual transmitter are inherently synchronized.

The concept is similar if the reflection happens at a punctual scatterer. This situation is depicted in Fig. 3. Now, if the receiver moves through this scenario, the signals scattered from this punctual scatterer always seem to be emerging from the scatterer itself. Hence, we obtain a virtual transmitter vTx again. However, the virtual transmitter has an additional clock offset $\tau_{0}$, which is the Euclidean distance between the physical and the virtual transmitter divided by the speed of light. This clock offset is constant if the positions of the physical and the virtual transmitter are static, which is our assumption.

The concepts of single reflections and single scatterings can be generalized in a straightforward manner to situations where a signal from a physical transmitter undergoes multiple reflections and scatterings [5].

\section{B. Recursive Bayesian Estimation}

Recursive Bayesian estimation allows in general for estimating a probability density function (PDF) of a state vector $\mathbf{x}$ recursively over time based on a mathematical model of the ongoing process and incoming measurements [8]. It is assumed that $\mathbf{x}$ follows a hidden Markov model, which cannot be observed directly. The evolution of the state $\mathbf{x}$ with time is modeled as

$$
\mathbf{x}_{k}=\mathbf{f}_{k}\left(\mathbf{x}_{k-1}, \mathbf{v}_{k-1}\right)
$$

where $\mathbf{x}_{k}$ and $\mathbf{x}_{k-1}$ are the state vector at time steps $k$ respectively $k-1, \mathbf{f}_{k}$ is a known function, and $\mathbf{v}_{k}$ is the realization of a process noise sequence at time step $k$. Assuming this model of the process to be available in a probabilistic form, we can get prior information on a successive time step. This means that we can calculate the probability for the state in the next time step, which is

$$
\mathrm{p}\left(\mathbf{x}_{k} \mid \mathbf{x}_{k-1}\right) \text {. }
$$

Equation Eq. (1) describes the so-called movement model or state transition of the process. Similarly to Eq. (1), measurements of the state can be modeled as

$$
\mathbf{z}_{k}=\mathbf{h}_{k}\left(\mathbf{x}_{k}, \mathbf{n}_{k}\right)
$$

where $\mathbf{z}_{k}$ is the measurement, $\mathbf{h}_{k}$ is a known function, and $\mathbf{n}_{k}$ is one realization of a measurement noise sequence at time step $k$. The likelihood, i.e., the probability for the measurement given the current state, is

$$
\mathrm{p}\left(\mathbf{z}_{k} \mid \mathbf{x}_{k}\right) \text {. }
$$

The goal is to estimate the states $\mathbf{x}_{k}$ given all $k$ conducted measurements $\mathbf{z}_{1: k}$, or more precisely, to estimate the posterior PDF $\mathrm{p}\left(\mathbf{x}_{k} \mid \mathbf{z}_{1: k}\right)$. This problem is approached recursively in two stages, namely prediction and update.

Some calculations [9] yield that the prediction of the next state $\mathbf{x}_{k}$ can be calculated by

$$
\mathrm{p}\left(\mathbf{x}_{k} \mid \mathbf{z}_{1: k-1}\right)=\int \underbrace{\mathrm{p}\left(\mathbf{x}_{k} \mid \mathbf{x}_{k-1}\right)}_{\text {Eq. [2 }} \underbrace{\mathrm{p}\left(\mathbf{x}_{k-1} \mid \mathbf{z}_{1: k-1}\right)}_{\text {previous update }} d \mathbf{x}_{k-1},
$$

and the update is given by

$$
\mathrm{p}\left(\mathbf{x}_{k} \mid \mathbf{z}_{1: k}\right)=\frac{1}{\alpha_{k}} \underbrace{\mathrm{p}\left(\mathbf{z}_{k} \mid \mathbf{x}_{k}\right)}_{\text {Eq. (4) }} \underbrace{\mathrm{p}\left(\mathbf{x}_{k} \mid \mathbf{z}_{1: k-1}\right)}_{\text {previous prediction }},
$$

where $\alpha_{k}=\int \mathrm{p}\left(\mathbf{z}_{k} \mid \mathbf{x}_{k}\right) \mathrm{p}\left(\mathbf{x}_{k} \mid \mathbf{z}_{1: k-1}\right) d \mathbf{x}_{k}$ is a constant term. However, the integrals in Eq. (5) and Eq. (6) can in general not be solved analytically.

\section{Estimation of the Parameters of MPCs}

As the mobile terminal moves through the scenario, it receives signals that are periodically transmitted by one or more physical transmitters. We assume the signals from different physical transmitters to be separated in frequency. Hence, this subsection refers to estimating the parameters of MPCs for only one physical transmitter. Due to reflections and scattering of the transmitted signal, the signal arrives at the antenna of the mobile terminal via multiple paths. This allows for modeling the received signal at time step $k$ as the superposition of $L_{k}$ transmit signals $s(\tau)$ of complex amplitudes $\alpha_{l, k}$ with delays $\tau_{l, k}$, namely 


$$
y_{k}(\tau)=\sum_{l=0}^{L_{k}-1} \alpha_{l, k} s\left(\tau-\tau_{l, k}\right)+n_{k}(\tau) .
$$

The term $n_{k}(\tau)$ denotes additive white Gaussian noise (AWGN). For every time step $k$ at time $t_{k}$, a snapshot of the received signal is sampled and recorded. For every snapshot, the Space-Alternating Generalized ExpectationMaximization (SAGE) algorithm [10] is used to estimate the complex amplitude $\alpha_{l, k}$ and delay $\tau_{l, k}$ for each MPC. The SAGE algorithm jointly estimates these parameters, as it is an extension of the Expectation-Maximization (EM) algorithm [11] approximating the maximum likelihood solution. As the receiver moves through the scenario, the parameters of the MPCs will change over time. We use the Kalman enhanced super resolution tracking (KEST) algorithm as described in [12] in order to track the delay, the absolute value of the magnitude, and the phase of each MPC and to describe their evolution as the mobile terminal travels through the scenario. As the name suggests, the tracking is done by a Kalman filter. The Kalman filter is a special case of recursive Bayesian estimation, where the functions $\mathbf{f}_{k}$ in the movement model and $\mathbf{h}_{k}$ in the measurement model in (1) respectively (3) are linear in the state $\mathbf{x}_{k}$, and $\mathbf{n}_{k}$ and $\mathbf{v}_{k}$ are samples of AWGN with zero mean. Then, it can be shown that both the PDFs Eq. (2) and Eq. (4), and the integrals in Eq. (5) and Eq. (6) are Gaussian densities and can be parameterized by a mean and a covariance. Therefore, Eq. (5) and Eq. (6) can be solved analytically and efficiently in the recursive estimation process. The state at time step $k$ consists of the delays and complex amplitudes of all current MPCs, i.e.,

$\left[\ldots, \quad \tau_{i, k}, \quad a_{i, k}, \quad \arg \left\{\alpha_{i, k}\right\}, \quad \ldots\right]^{\mathrm{T}}, \quad i=0, \ldots, L_{k}-1$,

where $a_{i, k}=\left|\alpha_{i, k}\right|$ is the absolute value and $\arg \left\{\alpha_{i, k}\right\}$ is the phase of $\alpha_{i, k}$. Since no prior information on the evolution of the MPCs is assumed, the movement model is implemented as a simple random walk model. During the receiver motion, MPCs might arise and vanish depending on the position of the mobile terminal. Hence, the KEST algorithm also estimates and keeps track of the number $L_{k}$ of MPCs. For the initialization of their number in the first time step, i.e., $L_{0}$, the Bayesian information criterion rule as in [13] is applied.

\section{Position Estimation}

The estimates of the KEST algorithm are used to track the mobile terminal and to determine the positions of the physical and virtual transmitters. For the mobile terminal, we estimate both the coordinates and the velocity in two dimensions each for each time step $k$, i.e.,

$$
\mathbf{x}_{\mathrm{MT}}(k)=\left[\begin{array}{llll}
x_{\mathrm{MT}}(k) & y_{\mathrm{MT}}(k) & v_{x, \mathrm{MT}}(k) & v_{y, \mathrm{MT}}(k)
\end{array}\right]^{\mathrm{T}} .
$$

As the virtual transmitters are assumed to be static, only their position, i.e., $x_{l}$ and $y_{l}$, and their delay offset $\tau_{0, l}$ have to be estimated. For the $l^{\text {th }}$ virtual transmitter, this is

$$
\mathbf{x}_{\mathrm{VT}, l}=\left[\begin{array}{lll}
x_{l} & y_{l} & \tau_{0, l}
\end{array}\right]^{\mathrm{T}} .
$$

The entire state to be estimated at a time step $k$ is therefore

$$
\mathbf{x}_{k}=\left[\begin{array}{llll}
\mathbf{x}_{\mathrm{MT}}(k)^{T} & \mathbf{x}_{\mathrm{VT}, 0}^{T} & \cdots & \mathbf{x}_{\mathrm{VT}, L_{k}-1}^{T}
\end{array}\right]^{\mathrm{T}} .
$$

Since we do not assume synchronization between the physical transmitters and the mobile terminal, we cannot use the KEST delay estimates, i.e., the delays of the single MPCs, directly. Instead, we calculate the time differences of arrival (TDoAs) between the incoming MPCs. The true propagation time between the $l^{\text {th }}$ transmitter and the mobile terminal can be calculated as

$$
d_{\mathrm{MT}, l}(k)=\frac{1}{c_{0}} \sqrt{\left(x_{\mathrm{MT}}(k)-x_{l}\right)^{2}+\left(y_{\mathrm{MT}}(k)-y_{l}\right)^{2}}+\tau_{0, l},
$$

where $c_{0}$ denotes the speed of light. Hence, the TDoA between two signals from the $l^{\text {th }}$ and $m^{\text {th }}$ virtual transmitter is

$$
\Delta d_{l, m}(k)=d_{\mathrm{MT}, m}(k)-d_{\mathrm{MT}, l}(k) .
$$

This makes the measurement function $\mathbf{h}_{k}$ in Eq. (3) nonlinear in the state. Hence, the standard Kalman filter can not be used, and the optimal Bayesian solution is intractable. Instead, we use a Monte Carlo method, namely a sequential importance resampling (SIR) particle filter [9]. The idea of a particle filter is to estimate the PDF of a state using an approximation of the optimal Bayesian solution by means of a large set of tuples, so called particles. Each of the particles consists of a point in the state space and an associated weight. In contrast to Kalman filters, this approximation enables particle filters to handle also nonlinear filtering problems in the presence of arbitrary, not necessarily Gaussian noise.

\section{E. 3GPP-LTE Positioning Reference Signals}

As mentioned in the introduction, the 3GPP-LTE PRSs are optional signals. They are spread over the orthogonal frequency-division multiplexing (OFDM) frame structure in blocks of fixed length, which are repeated periodically. The possible length of these blocks is predefined by the LTE standard and ranges from 1 to 6 consecutive subframes. It is denoted by $N_{\text {PRS }}$. The periodicity of the blocks is predefined by the LTE standard as well. It is denoted by $T_{\mathrm{PRS}}$, and its possible values range from 160 to 1280 subframes. Also, the bandwidth of the PRSs is variable, with possible values ranging from $1.4 \mathrm{MHz}$ up to $20 \mathrm{MHz}$, which is also the maximum bandwidth of a 3GPP-LTE system. The PRSs are pseudo-random sequences. They have a cell-specific frequency shift ranging from 0 to 5 subcarriers. i.e., there can be no more than six LTE base stations without interfering PRSs.

\section{Measurements}

\section{A. Measurement Setup}

Fig. 4 gives an overview over the measurement scenario. The measurements were performed in front of a hangar with metal doors as depicted in the figure. A receiver was carried by 


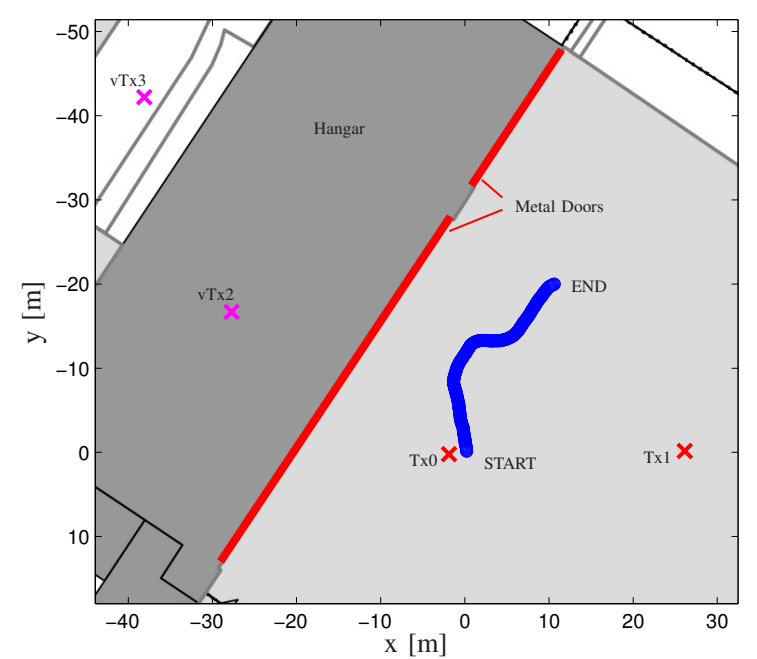

Fig. 4. The measurements were taken in front of a hangar with metal doors. The mobile terminal was moving along the blue track. Tx0 and Tx1 denote the positions of the LTE base stations.

TABLE I

Parameters of THE USED 3GPP-LTE Signals

\begin{tabular}{ll}
\hline Parameter & Value \\
\hline RF carrier frequency & $2.46 \mathrm{GHz}$ \\
3GPP-LTE bandwidth & $20 \mathrm{MHz}$ \\
3GPP-LTE PRS bandwidth & $20 \mathrm{MHz}$ \\
$N_{\text {PRS }}$ & 6 \\
\hline
\end{tabular}

a pedestrian acting as the mobile terminal along the blue track of length 30.22 meters, where the labels START and END mark its start respectively end position. There are two 3GPPLTE base stations, i.e., physical transmitters, indicated by the red crosses with labels Tx 0 and Tx1. The magenta crosses with labels vTx2 and vTx3 mark the positions where we expect to find virtual transmitters for the physical transmitters Tx0 respectively $\mathrm{Tx} 1$, if their signals are reflected by the metal doors of the hangar. Hence, these points do not have any physical meaning. The pedestrian walked along the track with an average speed of 0.322 meters per second. The ground truth of the positions of the mobile terminal and the physical transmitters was obtained by means of a tachymeter with centimeter accuracy. The receiver recorded a snapshot of the 3GPP-LTE signal every 70 milliseconds.

An overview of the used hardware is given in Fig. 5. We used a Rohde \& Schwarz SMW200A Vector Signal Generator to generate 3GPP-LTE signals. This signal generator has two radio frequency (RF) outputs that fed the two transmit antennas of the physical transmitters. At the mobile terminal, the signals were received, sampled, and recorded for postprocessing by a data grabber. Table \ specifies important parameters of the used 3GPP-LTE signals.

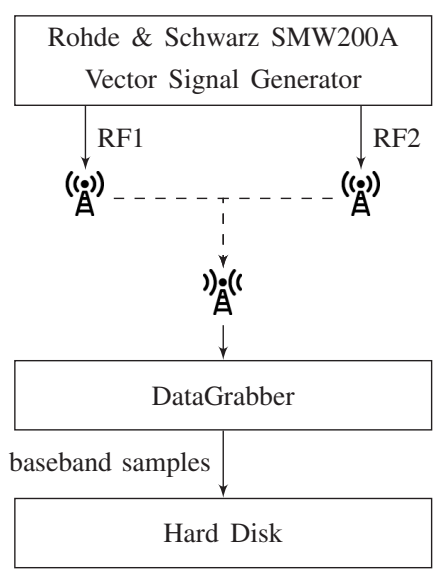

Fig. 5. Overview of the measurement setup: A signal generator generates the 3GPP-LTE signals that are transmitted by the two physical transmitters. A data grabber samples the signal received by the antenna at the mobile terminal.

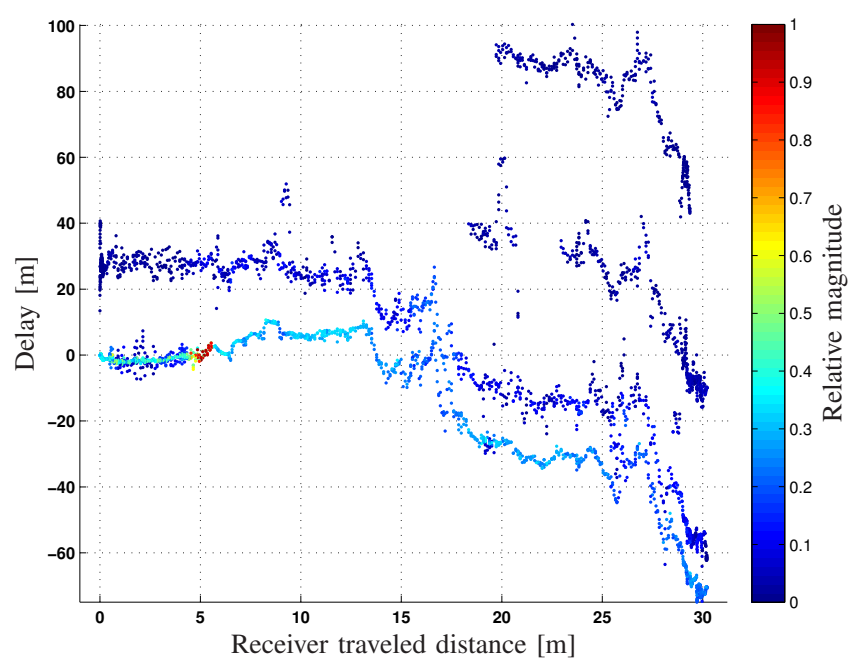

Fig. 6. Results of the KEST algorithm for the physical transmitter Tx0. The estimated delay times the speed of light is plotted over the receiver traveled distance. The color indicates the normalized, estimated amplitudes in linear domain.

\section{B. Measurement Results}

The results of the KEST algorithm for the physical transmitters Tx0 and Tx1 are plotted in Fig. 6 respectively Fig. 7 They show the delays and amplitudes of the estimated MPCs over the distance the receiver has traveled. The delays are multiplied by the speed of light. Note that the actual delays are not of interest, since the receiver is not synchronized to the physical transmitters. We only use the relative delays between the MPCs for each snapshot. The color shows the normalized absolute values of the amplitudes of the corresponding MPC as estimated by the KEST algorithm. They are normalized and given in linear domain.

For position estimation, we use three TDoAs as measurement input for the particle filter: the first one is the TDoA between the strongest paths of the physical transmitters Tx0 and Tx1. In addition, for each of the two physical transmitters, we use the TDoAs between the strongest and second strongest 


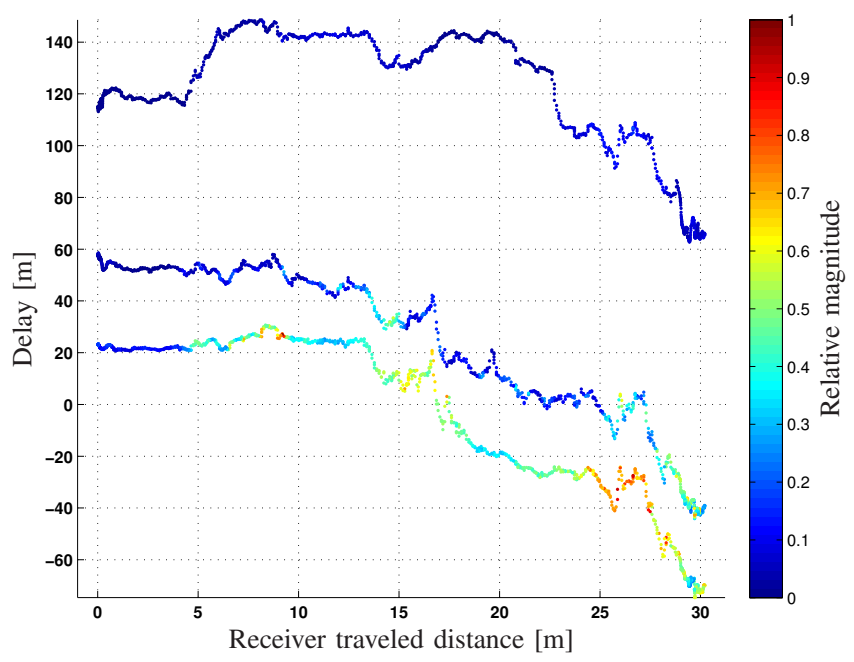

Fig. 7. Results of the KEST algorithm for the physical transmitter Tx1. The estimated delay times the speed of light is plotted over the receiver traveled distance. The color indicates the normalized, estimated amplitudes in linear domain.

path.

We use heading information from an IMU in the position estimation step in order to improve the tracking of the mobile terminal. This prior information is fed into the particle filter in the form of a movement model, i.e., it is covered by the function $\mathbf{f}_{k}$ from Eq. (1). However, the IMU heading provides only reliable information on the direction of the movement of the mobile terminal. As we do not assume any prior information for its speed, we incorporate a random walk model for the absolute value of the velocity the mobile terminal is moving at.

The root mean square error (RMSE) for positioning is plotted in Fig. 8. The positioning error for the mobile terminal is always below 1.8 meters, in $90 \%$ of the cases, it is below 1.25 meters. The position of the physical and virtual transmitters is unknown in the beginning, and the particles are spread over the area. In the beginning, the positioning error for the physical transmitters Tx 0 and Tx 1 is in the order of 15 to 20 meters. After about 20 meters of traveled distance, many hypotheses for the position of the physical transmitters are rejected, which leads to some fluctuations in the positioning error. The positioning error is then around 3.5 meters for both Tx0 and Tx1. We assume the virtual transmitters to arise due to reflections at the metal doors of the hangar as depicted in Fig. 4 and hence, that these virtual transmitters are in the positions of vTx2 and vTx3. The RMS positioning error for the virtual transmitters is therefore calculated assuming these positions as the true ones. Their positioning error decreases from ca. 20 meters in the beginning down to ca. 3.3 meters for vTx2 and to ca. 5.3 meters for vTx3.

\section{Conclusion}

The main contribution of this paper is to show that multipath-assisted positioning using 3GPP-LTE signals is possible despite of the constraint of their relatively low maximum

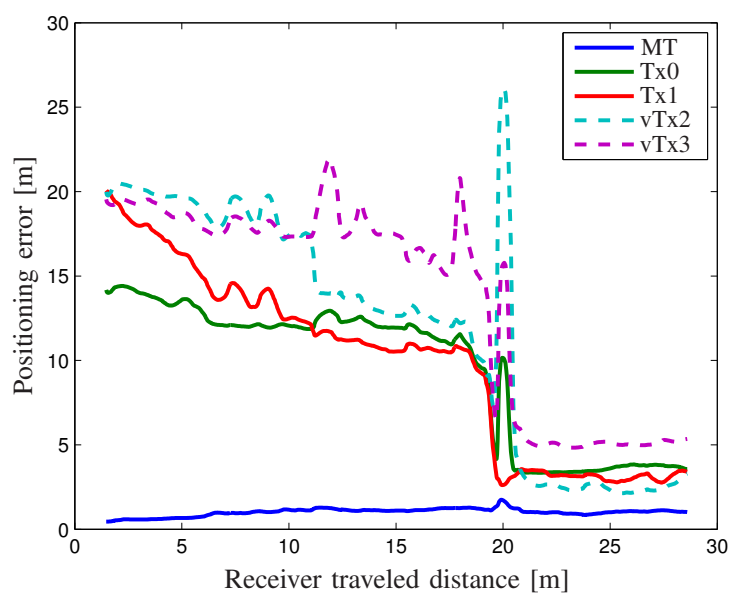

Fig. 8. The RMSE for positioning the mobile terminal (MT) and the physical and virtual transmitters. For the virtual transmitters vTx2 and vTx3, we assume that they arise due to reflections of the signals from Tx 0 respectively vTx1 at the metal doors as depicted in Fig. 4

bandwidth of $20 \mathrm{MHz}$. Therefore, we performed outdoor measurements. In a first step, we tracked the parameters of MPCs arriving at the receiver by means of a Kalman filter. We used these estimates together with heading information from an IMU as input for the Channel-SLAM algorithm. This allows for simultaneously tracking a mobile terminal moving through the scenario, and determining the positions of virtual or physical transmitters. The evaluations show that the RMSE for the mobile terminal position is in the order of 1 meter most of the time. For the transmitters, the RMSE settles down to ca. 3.3 respectively 5.3 meters for the virtual transmitters, and to ca. 3.5 meters for the physical transmitters.

\section{REFERENCES}

[1] P. Meissner and K. Witrisal, "Multipath-assisted single-anchor indoor localization in an office environment," in Systems, Signals and Image Processing (IWSSIP), 2012 19th International Conference on, Apr. 2012, pp. 22-25.

[2] P. Setlur, G. Smith, F. Ahmad, and M. Amin, "Target localization with a single sensor via multipath exploitation," Aerospace and Electronic Systems, IEEE Transactions on, vol. 48, no. 3, pp. 1996-2014, Jul. 2012.

[3] M. Ulmschneider, C. Gentner, S. Ache, and A. Roessler, "Multipath assisted positioning with band-limited signals in an urban environment," in Proceedings of the 28th International Technical Meeting of The Satellite Division of the Institute of Navigation (ION GNSS+ 2015), Tampa, FL, USA, Sep. 2015, pp. 2329-2334.

[4] A. O'connor, P. Setlur, and N. Devroye, "Single-sensor rf emitter localization based on multipath exploitation," Aerospace and Electronic Systems, IEEE Transactions on, vol. 51, no. 3, pp. 1635-1651, July 2015.

[5] C. Gentner, T. Jost, and A. Dammann, "Indoor positioning using time difference of arrival between multipath components," in IEEE IPIN, Montbeliard, France, Oct. 2013.

[6] C. Gentner, R. Pöhlmann, M. Ulmschneider, T. Jost, and A. Dammann, "Multipath assisted positioning for pedestrians," in Proceedings of the ION GNSS+, Tampa, FL, USA, Sep. 2015.

[7] G. T. 36.211, "Evolved universal terrestrial radio access (e-utra); physical channels and modulation," Oct. 2012, v11.0.0.

[8] S. Kay, Fundamentals of Statistical Signal Processing: Estimation Theory, ser. Fundamentals of Statistical Signal Processing. PrenticeHall PTR, 1998, no. Bd. 1. 
[9] M. Arulampalam, S. Maskell, N. Gordon, and T. Clapp, "A tutorial on particle filters for online nonlinear/non-gaussian bayesian tracking," Trans. Sig. Proc., vol. 50, no. 2, pp. 174-188, Feb. 2002.

[10] B. Fleury, M. Tschudin, R. Heddergott, D. Dahlhaus, and K. Pedersen, "Channel parameter estimation in mobile radio environments using the sage algorithm," IEEE JSAC for Wireless Communications Series, vol. 17, no. 3, pp. 434-450, Mar. 1999.

[11] A. P. Dempster, N. M. Laird, and D. B. Rubin, "Maximum likelihood from incomplete data via the em algorithm," Journal of the Royal
Statistical Society. Series B (Methodological), vol. 39, no. 1, pp. 1-38, 1977.

[12] T. Jost, W. Wang, U. Fiebig, and F. Perez-Fontan, "Detection and tracking of mobile propagation channel paths," Antennas and Propagation, IEEE Transactions on, vol. 60, no. 10, pp. 4875-4883, Oct. 2012.

[13] P. Stoica and Y. Selen, "Model-order selection: a review of information criterion rules," Signal Processing Magazine, IEEE, vol. 21, no. 4, pp. 36-47, Jul. 2004 